\title{
Position determination of the Chang'e 3 lander with geodetic VLBI
}

\author{
Grzegorz Klopotek ${ }^{1 *}$ (1) Thomas Hobiger ${ }^{2}$, Rüdiger Haas ${ }^{1}$, Frédéric Jaron ${ }^{3}$, Laura La Porta ${ }^{3,4}$, Axel Nothnagel ${ }^{3}$, \\ Zhongkai Zhang ${ }^{3}$, Songtao Han ${ }^{5}$, Alexander Neidhardt ${ }^{6}$ and Christian Plötz ${ }^{7}$
}

\begin{abstract}
We present results from the analysis of observations of the Chang'e 3 lander using geodetic Very Long Baseline Interferometry. The applied processing strategy as well as the limiting factors to our approach is discussed. We highlight the current precision of such observations and the accuracy of the estimated lunar-based parameters, i.e., the lunar lander's Moon-fixed coordinates. Our result for the position of the lander is $44.12193^{\circ} \mathrm{N},-19.51159^{\circ} \mathrm{E}$ and $-2637.3 \mathrm{~m}$, with horizontal position uncertainties on the lunar surface of $8.9 \mathrm{~m}$ and $4.5 \mathrm{~m}$ in latitude and longitude, respectively. This result is in good agreement with the position derived from images taken by the Narrow Angle Camera of the Lunar Reconnaissance Orbiter. Finally, we discuss potential improvements to our approach, which could be used to apply the presented concept to high-precision lunar positioning and studies of the Moon.
\end{abstract}

Keywords: Moon, Geodetic Very Long Baseline Interferometry, c5++, Chang'e 3, OCEL

\section{Introduction}

Very Long Baseline Interferometry (VLBI) has a long history of observations of interplanetary spacecrafts (Duev et al. 2012; Jones et al. 2015) where targets and nearby reference radio sources are observed in an alternating order. Such projects aim to improve planetary ephemerides or conduct tests of general relativity. Progress in space sciences and a continuous interest in the exploration of the Solar System have also motivated numerous missions with the aim to study the Moon (Kato et al. 2008; Elphic et al. 2014). A recent example is the Chinese Chang'e 3 (CE-3) mission, started in the end of 2013 with the deployment of a lander and a rover at the northwest part of the visible side of the Moon (Li et al. 2015). The lander is equipped with a digital communication channel at $\mathrm{X}$ band with the capability to transmit Differential One-way Ranging (DOR) tones. Since the successful landing of the CE-3 probes, various efforts have been made to determine their relative and absolute positions. The former concerns positioning of the rover w.r.t.

\footnotetext{
*Correspondence: grzegorz.klopotek@chalmers.se

${ }^{1}$ Department of Space, Earth and Environment, Onsala Space

Observatory, Chalmers University of Technology, Onsala, Sweden

Full list of author information is available at the end of the article
}

the lander with meter-level accuracy (Zhou et al. 2015). Examples for the latter are utilization of photogrammetry images taken from a Chinese orbiter (Liu et al. 2015b) or by the Lunar Reconnaissance Orbiter (LRO) Narrow Angle Camera (NAC) (Tooley et al. 2010), leading to position estimates with an accuracy of better than $20 \mathrm{~m}$ (Liu et al. 2015a). The position determination from the combination of Unified X-band (UXB) (range and rangerate measurements) and VLBI observations, carried out with Chinese telescopes and lasting a few hours, led to uncertainties of a few tens of meters (Li et al. 2014; Cao et al. 2016). Differential VLBI observations of lunar targets with nearby calibrator radio sources might be problematic due to the angular size of the Moon, implying a few-degree angular separations and propagating, in this case, position uncertainties of reference sources into lunar-based parameters, proportionally to the target-calibrator distance on the sky.

The CE-3 mission created also a great opportunity to observe the lander in an absolute sense, i.e., with standard geodetic VLBI. First observations were performed already in 2014 with a pair of European telescopes (Klopotek et al. 2017a). Subsequently, the International VLBI Service for Geodesy and Astrometry (IVS) (Nothnagel et al. 2017) Observing Program Committee approved 
observations of the lander with an increased number of participating telescopes. This effort was realized within the OCEL (Observing the Chang'e 3 Lander with VLBI) program where geodetic VLBI observations of the lander's DOR tones were carried out in 2014, 2015 and 2016 during twelve 24-h sessions and with a global network of VLBI telescopes (Haas et al. 2017).

In the following sections, the concept of geodetic VLBI for observations of artificial radio sources on the lunar surface is described and the results from the analysis of OCEL sessions are presented. In addition, we provide information on the current precision of such new observations as well as the accuracy of the determined position of the CE-3 lander. The obtained values are compared with results from independent studies as well as our Monte Carlo simulations. We also provide information on the limiting factors of the presented concept and discuss the prospective possibilities for future improvements.

\section{Data and methods}

Geodetic VLBI is a space-geodetic technique utilizing observations of very distant natural radio sources, e.g., quasi-stellar objects, commonly referred to as quasars. A suitable geodetic radio source is characterized by a compact (point-like) structure, high flux density, and a well-established celestial position. The basic observable used in geodetic VLBI is the time difference of quasar signal reception between two radio telescopes forming a baseline. Such observations are carried out nowadays at X band and S band (Sovers et al. 1998). Through the use of many quasars and a global network of VLBI telescopes, valuable information on the orientation and rotation of the Earth w.r.t. the inertial reference frame, defined by a catalog of well-defined natural radio sources, can be accessed (Fey et al. 2009).

\section{Combination of lunar and quasar observations}

The concept to schedule the OCEL sessions is described in detail by Haas et al. (2017) along with extensive information on the CE-3 signal characteristics as well as the frequency setups used during those experiments. Provided that the Moon was visible during the 24-h session by at least two of the globally distributed telescopes in the network, a complete 24-h session was separated into 30-minute blocks with observations. These blocks were arranged in an alternating order and included either solely quasar observations (quasar blocks) or solely observations of the CE-3 lander with all the telescopes that could see the Moon (lunar blocks). This is illustrated in Fig. 1. Additionally, the lunar blocks included also quasar observations for telescopes with no visibility of the Moon. This scheduling concept allows to determine the behavior of hydrogen masers (referred to as station clocks) and troposphere parameters from analyzing the quasar-only blocks. This information can subsequently be applied to the analysis of lunar observations in the form of corrections to the calculated VLBI delays at each observation epoch.

The analysis of the lunar data requires also compensation for propagation effects caused by the ionosphere as these observations were carried out using a single frequency band (X band). Such ionosphere corrections can be calculated from Global Ionospheric Maps (GIM) which are provided in the IONosphere Map EXchange Format (IONEX) (Schaer et al. 1996). For lunar X-band

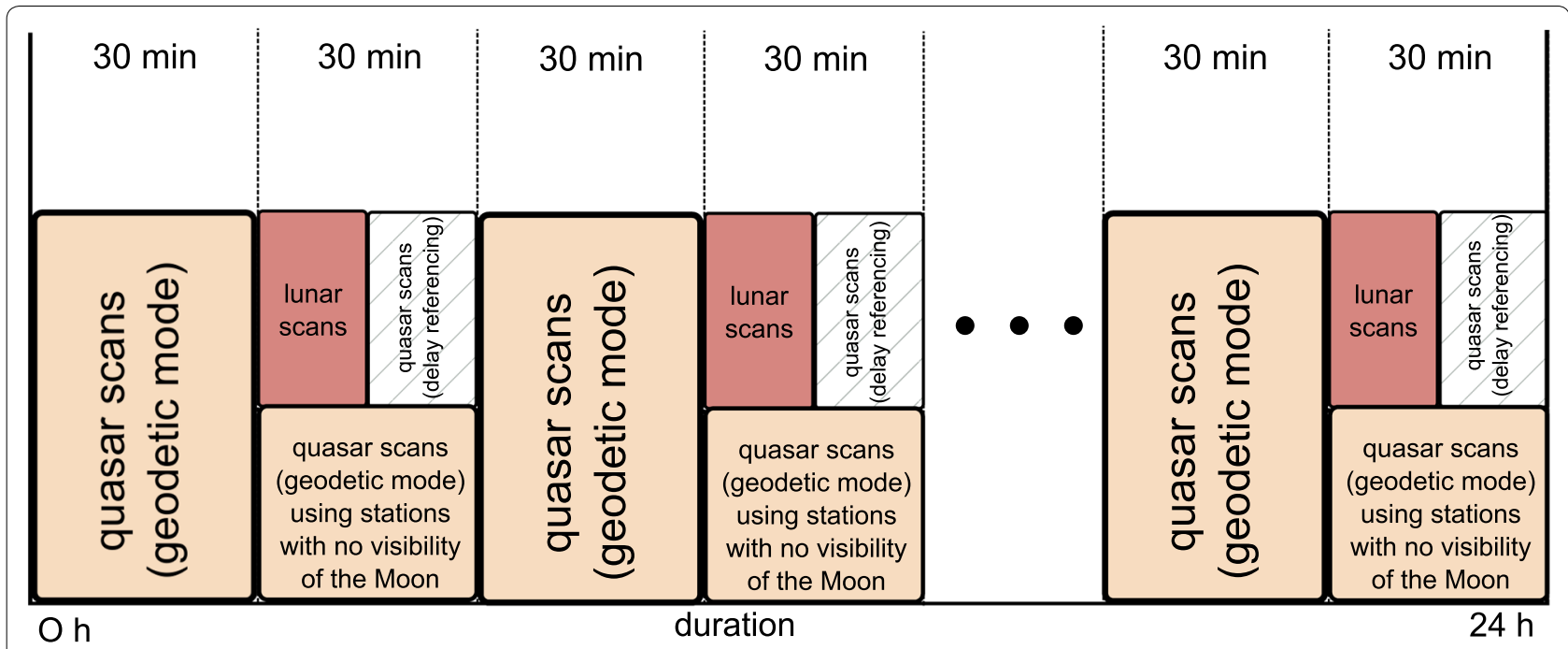

Fig. 1 Combination of quasar and lunar observations in the OCEL sessions. The delay-referencing scans are not part of this study 
observations, ionosphere delay corrections obtained in this way and applied to the VLBI analysis are expected to have an uncertainty of about $60 \mathrm{~mm}$ on a $8000-\mathrm{km}$ baseline (Sekido et al. 2003).

\section{Data processing}

The common geodetic VLBI processing chain consists of several stages including correlation, post-correlation extraction of geodetic VLBI observables, and final data analysis for the target parameter estimation. Due to the nature of DOR tones (four twenty-kHz-wide tones as opposed to the continuous spectrum of quasar signals) and the novelty of the proposed concept, the latter two stages had to be refined in order to handle lunar observations in a reliable manner. The processing strategy adopted here consists of four steps and is illustrated and briefly described in Fig. 2. In Step I, both the quasar and lunar observations were correlated with DiFX 2.40 (Deller et al. 2011) using 128 spectral channels per sub-band. Step II dealt with the extraction of geodetic VLBI observables (multi-band delays) for both lunar and quasar data. This was carried out using Fourfit (Lonsdale 1996), a software commonly used in the standard geodetic post-correlation analysis. The only difference between the processing of quasar and lunar data in this case is the utilization of the passband option of Fourfit, allowing to restrict the cross-power spectra to windows of one $\mathrm{MHz}$ for each of the four DOR tones, without including the carrier frequency. A quite similar approach, related to DOR tones and Fourfit, has been successfully applied to observations of Earth-orbiting satellites with geodetic VLBI (Hellerschmied et al. 2018). In Step III, quasar-only delays in S band and X band were analyzed with $\nu$ Solve (Bolotin et al. 2014) to resolve ambiguities and derive ionosphere delays. Step IV covered the target parameter estimation using c5++ (Hobiger et al. 2010), whose VLBI module has been recently extended with the possibility to utilize both quasar and lunar observations (Klopotek et al. 2018). Weighted least-squares adjustment in c5 ++ includes variance component estimation (Hobiger and Otsubo 2017) resulting in $\chi^{2} / v$ close to

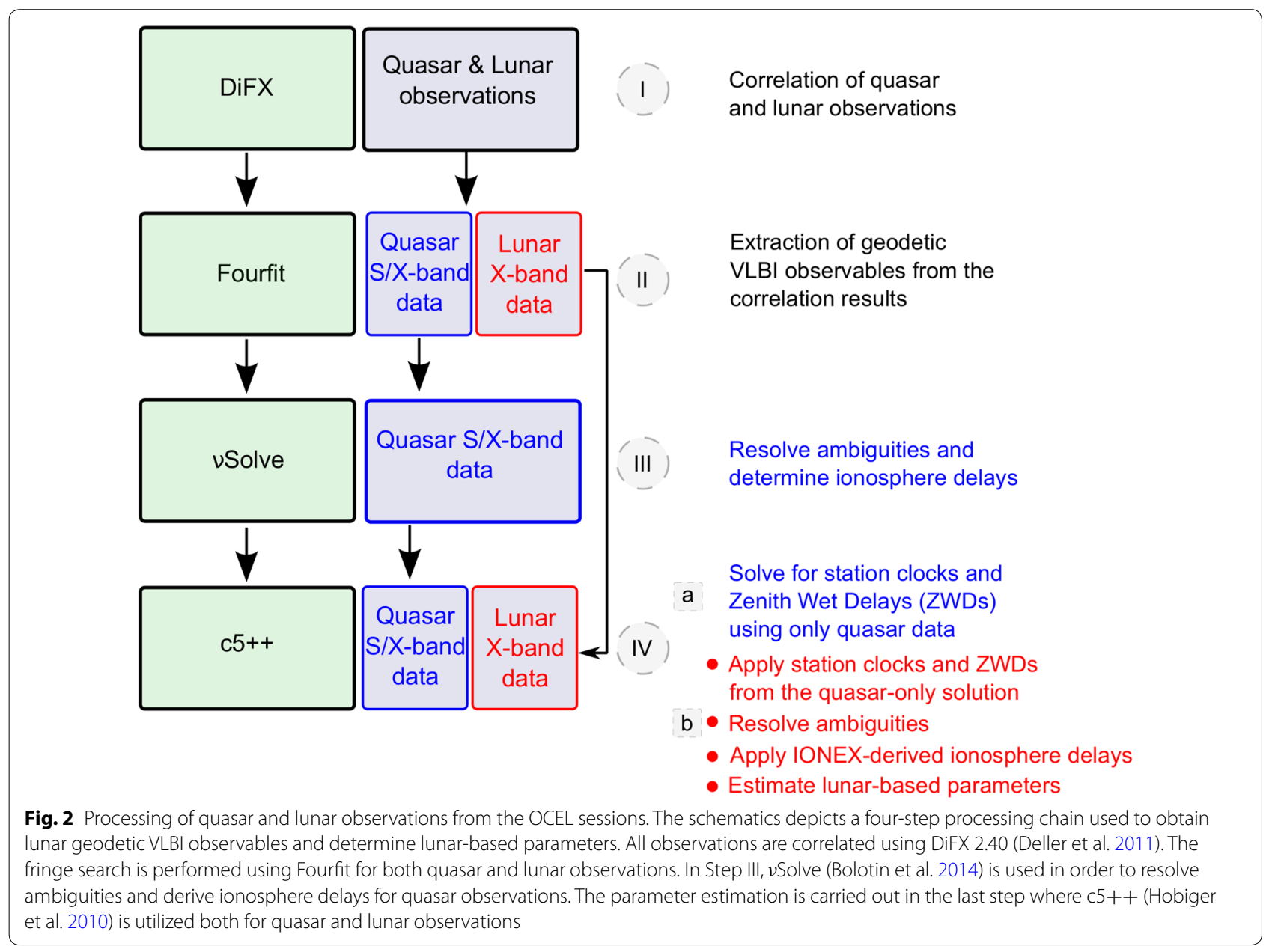


unity, leading to more realistic uncertainties of the estimated target parameters.

In the following, we focus on the results derived from the analysis of RD1510 and RD1601 sessions (referred to as OCEL08 and OCEL09). In a prestudy (Klopotek et al. 2017b) these two sessions were identified as the best-performing among the twelve OCEL sessions. The other sessions are characterized by a worse observing geometry and were affected by various problems experienced at the observation and correlation stages, thus preventing their inclusion into this study. This is highlighted in Table 1 based upon a representative group of sessions.

\section{VLBI delay modeling and parameter estimation}

When considering quasar observations, the geodetic VLBI analysis follows a well-established modeling approach (Petit and Luzum 2010). This, however, is not applicable to artificial radio sources on the Moon and the conventional VLBI delay model needs to be replaced with a model considered for targets at a finite distance (Duev et al. 2012) and applied both to correlation and data analysis (Klopotek et al. 2017a; Hellerschmied et al. 2018).

The position of the CE-3 lander was solved in lunar latitude and longitude $\left(\phi_{\text {lan }}, \lambda_{\text {lan }}\right)$ in the Moon-fixed (Mean-Earth) reference frame (Archinal et al. 2011) and height $\left(H_{\text {lan }}\right)$ w.r.t. a reference sphere with a radius

Table 1 Characteristics of OCEL sessions and problems encountered during the analysis, shown for the selected experiments

\begin{tabular}{|c|c|c|}
\hline Session & Quasar data & Lunar data \\
\hline \multirow[t]{4}{*}{ RD1405 (OCEL01) } & Stations used (8): Bd, Hh, Ho, Ny, On, Sh, Wz, Zc & (6): Bd, Ny, Ho, On, Wz, Zc \\
\hline & No. of usable/scheduled obs.: 2622/6585 & $301 / 1018$ \\
\hline & Mc scheduled, but not participated & Most obs. during the last $6 \mathrm{~h}$ \\
\hline & Ny participating during the first $10 \mathrm{~h}$ & \\
\hline \multirow[t]{5}{*}{ RD1506 (OCELO6) } & Stations used (1 1): Bd, Ft, Hh, Ho, Kk, Mt, Ny, On, Sh, Wz, Zc & (8): Bd, Ft, Hh, Ho, Kk, Mt, Sh, Wz \\
\hline & No. of usable/scheduled obs.: 5064/6912 & $520 / 870$ \\
\hline & Manual phase calibration applied to all stations & 19-h observation period \\
\hline & $\mathrm{RFI}$ at $\mathrm{S}$ band (Ft, Ho, Mt, Sh, Zc) & \\
\hline & Problems at $\mathrm{X}$ band ( $\mathrm{Ho}, \mathrm{Ny}, \mathrm{Sh})$ & \\
\hline \multirow[t]{5}{*}{ RD1510 (OCELO8) } & Stations used (10): Bd, Ft, Ho, Ht, Kk, Mt, Ny, On, Wz, Zc & (9): No On \\
\hline & No. of usable/scheduled obs.: 3523/6045 & $856 / 1524$ \\
\hline & Manual phase calibration (Kk) & 22-h obs. period \\
\hline & $\mathrm{RFI}$ at $\mathrm{S}$ band $(\mathrm{Ft}, \mathrm{Mt}, \mathrm{Zc})$ & \\
\hline & Problems at $\mathrm{X}$ band (Ft, $\mathrm{Ny})$ & \\
\hline \multirow[t]{5}{*}{ RD1601 (OCELO9) } & Stations used (10): Bd, Ft, Ho, Hh, Kk, Mt, Ny, On, Wz, Zc & All \\
\hline & No. of usable/scheduled obs.: 3791/6094 & $1302 / 1427$ \\
\hline & Manual phase calibration (Ho, Kk) & 24-h obs. period \\
\hline & $\mathrm{RFl}$ at $\mathrm{S}$ band $(\mathrm{Ft}, \mathrm{Ho}, \mathrm{Mt}, \mathrm{Zc})$ & \\
\hline & Problems at $\mathrm{X}$ band (Ny) & \\
\hline \multirow[t]{5}{*}{ RD1604 (OCEL10) } & Stations used (10): Bd, Ft, Hh, Ho, Mc, Ny, On, Sh, Wz, Zc & (7): Bd, Ft, Hh, Wz, On, Sh, Zc \\
\hline & No. of usable/scheduled obs.: 2617/7272 & $547 / 1629$ \\
\hline & Manual phase calibration (Ho, Mc) & Most obs. during the first $8 \mathrm{~h}$ \\
\hline & $\mathrm{RFI}$ at $\mathrm{S}$ band $(\mathrm{Zc}, \mathrm{Ft}, \mathrm{Mc})$ & \\
\hline & Poor data quality at Mc: not used & $\begin{array}{l}\text { Poor data quality at Mc: not } \\
\text { used }\end{array}$ \\
\hline \multirow[t]{6}{*}{ RD1613 (OCEL12) } & Stations used (9): Bd, Hh, Kk, Mt, Ny, On, Ft, Wz, Zc & (8): No Ft \\
\hline & No. of usable/scheduled obs.: 3022/5339 & $345 / 1355$ \\
\hline & No signal in the last four X-band channels at Ft, Mt and Ny & 16-h obs. period \\
\hline & $\mathrm{RFI}$ at $\mathrm{S}$ band (Ft, Mt, Zc) & \\
\hline & Manual phase calibration (Kk) & \\
\hline & Ho and Ur - not correlated & \\
\hline
\end{tabular}

The italic font indicates sessions used in this study. Two-letter telescope codes: BADARY (Bd), FORTLEZA (Ft), HARTRAO (Hh), HART15M (Ht), HOBART26 (Ho), KOKEE (Kk), KUNMING (Km), MATERA (Mt), MEDICINA (Mc), NYALES20 (Ny), ONSALA60 (On), SESHAN25 (Sh), URUMQI (Ur), WETTZELL (Wz), ZELENCHK (Zc) 
of $1737.4 \mathrm{~km}$. Thereby, the theoretical VLBI delay modeling relies on JPL DE 421 (Folkner et al. 2009) for calculating the barycentric coordinates of the CE-3 lander and it includes periodic changes of the lunar lander's position caused by the solid Moon tides. Due to the nature of VLBI observations, i.e., poor sensitivity in the line-of-sight direction, $H_{\text {lan }}$ needs to be constrained to a well known a priori value (with $\sigma= \pm 10 \mathrm{~m}$ ) as VLBIonly observations do not allow for a reliable decoupling of all position components of the lander (Klopotek et al. 2018). However, range (or range-rate) observations could be used in the future to avoid an incorrect a priori height of the lander biasing the horizontal estimates by introducing an additional position shift. In the analysis, the a priori position of the CE-3 lander was assumed to be $0^{\circ} \mathrm{N}, 0^{\circ} \mathrm{E}$ and $-2637.6 \mathrm{~m}$ where the latter corresponds to the value reported by Cao et al. (2016) . The initial horizontal position of the lander is obviously not correct, but it was used at the beginning of the iterative estimation and to test the correctness of our analysis approach. Station clocks and tropospheric parameters were estimated in the quasar-only solution in Step IVa (see Fig. 2) and used in the lunar-only solution in Step IVb. The estimated Zenith Wet Delays (ZWDs) from the quasar-only solution are in good agreement with the corresponding results from the analysis of observations from co-located GNSS sites. Besides the ZWDs, horizontal tropospheric gradients and station clocks, other Earth-based parameters such as VLBI telescope positions expressed in ITRF2014 (Altamimi et al. 2016) as well as Earth Orientation Parameters (EOP IERS 14 C04 series (Bizouard and Gambis 2018)) were not estimated but fixed to their a priori values. Information on the parameterization of the nuisance parameters is given in Table 2. Moreover, any uncertainty in the Vertical Total Electron Content (VTEC) values from GIM propagates into a possible difference between IONEX-derived and geodetic VLBIderived ionosphere delays as an elevation-dependent error. For quasar data, the delay uncertainties originating from GIM-based ionosphere corrections amount to

Table 2 Parameterization of the nuisance parameters, i.e., clock and troposphere models

\begin{tabular}{|c|c|c|}
\hline \multirow[t]{2}{*}{ Parameter } & \multicolumn{2}{|l|}{ Parameterization } \\
\hline & OCEL08 & OCELO9 \\
\hline ZWD & $3 \mathrm{~h}$ PWL & \\
\hline Trop. gradients & One north and east component & \\
\hline Station clocks & $2 \mathrm{hPWL}$ & \\
\hline Clock reference & WETTZELL & \\
\hline
\end{tabular}

PWL refers to the piece-wise linear offset about $0.11 \mathrm{~m}$ and $0.09 \mathrm{~m}$ for OCEL08 and OCEL09 for an $8000-\mathrm{km}$ baseline, respectively. This was addressed in Step IVb by a constrained estimation (with $\sigma= \pm 3$ TEC units) of the VTEC biases (one per station per $24 \mathrm{~h}$ ) along with the lander's position components. In the same step, one additional clock offset per station was estimated as a constant parameter in order to handle ambiguities, potential absolute timing issues (Hellerschmied et al. 2018) as well as to correct for an unknown constant offset caused by instrumental delays at X band. The latter is usually absorbed by the clock model in the geodetic parameter estimation in the case of dual-frequency quasar data (Sekido et al. 2003; Hobiger et al. 2006). The iterative least-squares solutions converged with the WRMS of residuals equal to $0.26 \mathrm{~m}$ and $0.29 \mathrm{~m}$ for OCEL08 and OCEL09, respectively. The median formal uncertainty (from Fourfit) of the utilized lunar observables amounts to about $0.07 \mathrm{~m}$ for both sessions.

\section{Results}

Based on OCEL08 and OCEL09 as well as the presented approach, the position of the CE-3 lander was determined to be $44.12193^{\circ} \mathrm{N},-19.51159^{\circ} \mathrm{E}$ and $-2637.3 \mathrm{~m}$, with the horizontal position uncertainties on the lunar surface of $8.9 \mathrm{~m}$ and $4.5 \mathrm{~m}$ for $\phi_{\text {lan }}$ and $\lambda_{\text {lan }}$, respectively. These coordinates represent the weighted mean position of the lander computed based upon individual estimates from OCEL08 and OCEL09. In terms of the $1-\sigma$ horizontal position error ellipse, the obtained uncertainties can be translated to $3.0 \mathrm{~m}$ and $10.5 \mathrm{~m}$ for the semi-minor and semi-major axes, respectively.

Apart from real observations, a set of Monte Carlo simulations with the same parameterization and observing geometry as in the analysis of OCEL08 and OCEL09 was also carried out in order to validate the uncertainty of the obtained position estimates and quantify the impact of potential systematic effects. In Monte Carlo simulations, a geodetic VLBI observable comprises the calculated VLBI delay and contributions from three major error sources, i.e., tropospheric turbulence, reference clocks and thermal noise (Klopotek et al. 2018). The precision of simulated VLBI observations of the lander can be controlled with a baseline (Gaussian) noise, generated in our case with the standard deviation of the Gaussian random number generator set to $0.20 \mathrm{~m}$ and $0.24 \mathrm{~m}$ in order to obtain the post-fit WRMS of residuals derived from the analysis of OCEL08 and OCEL09. The analysis of both simulated OCEL observing geometries provided horizontal position error ellipses with a similar shape, orientation and semi-major axes, but with semi-minor axes smaller at least by a factor of two, compared to the analysis of real observations. This indicates the presence of 
some unidentified effects introducing additional uncertainty mostly in the longitudinal direction.

The obtained coordinates were also compared with results from other studies where different approaches were used to derive the position of the Chang'e 3 lander, see Fig. 3.

\section{Comparison to previous results}

The position estimates derived from OCEL sessions are close to the one stated by Liu et al. (2015a) (referred to as LRO NAC), see Fig. 3. On the lunar surface, the difference between our results and LRO NAC amounts to about $6 \mathrm{~m}$. The shape and magnitude of the error ellipses related to OCEL sessions (including our Monte Carlo simulations) can be attributed mostly to the contribution of additional clock offsets determined along with the lander's coordinates. These two groups of parameters are highly correlated (with Pearson correlation coefficients of up to 0.75 ) and most of the position uncertainty in the north-west direction can be related to this effect. Besides the observation noise and technique-specific error sources such as tropospheric turbulence or ionosphere, one needs to keep in mind that the difference between the position of the lander from LRO NAC and the location of the antenna (and its phase center, estimated and referred to in our case) that transmits the DOR signals is not well known and was neglected in our study as the present measurement sensitivity prevents identification of this effect.

One of the factors contributing to the different uncertainty levels between our estimates and the results stated by $\mathrm{Li}$ et al. (2014) (referred to as VLBI+UXB) can be related to the observing geometry and the session duration. Li et al. (2014) used only a few hours of observations with only Chinese telescopes. In the case of $24-\mathrm{h}$ OCEL sessions, intercontinental baselines were useful for

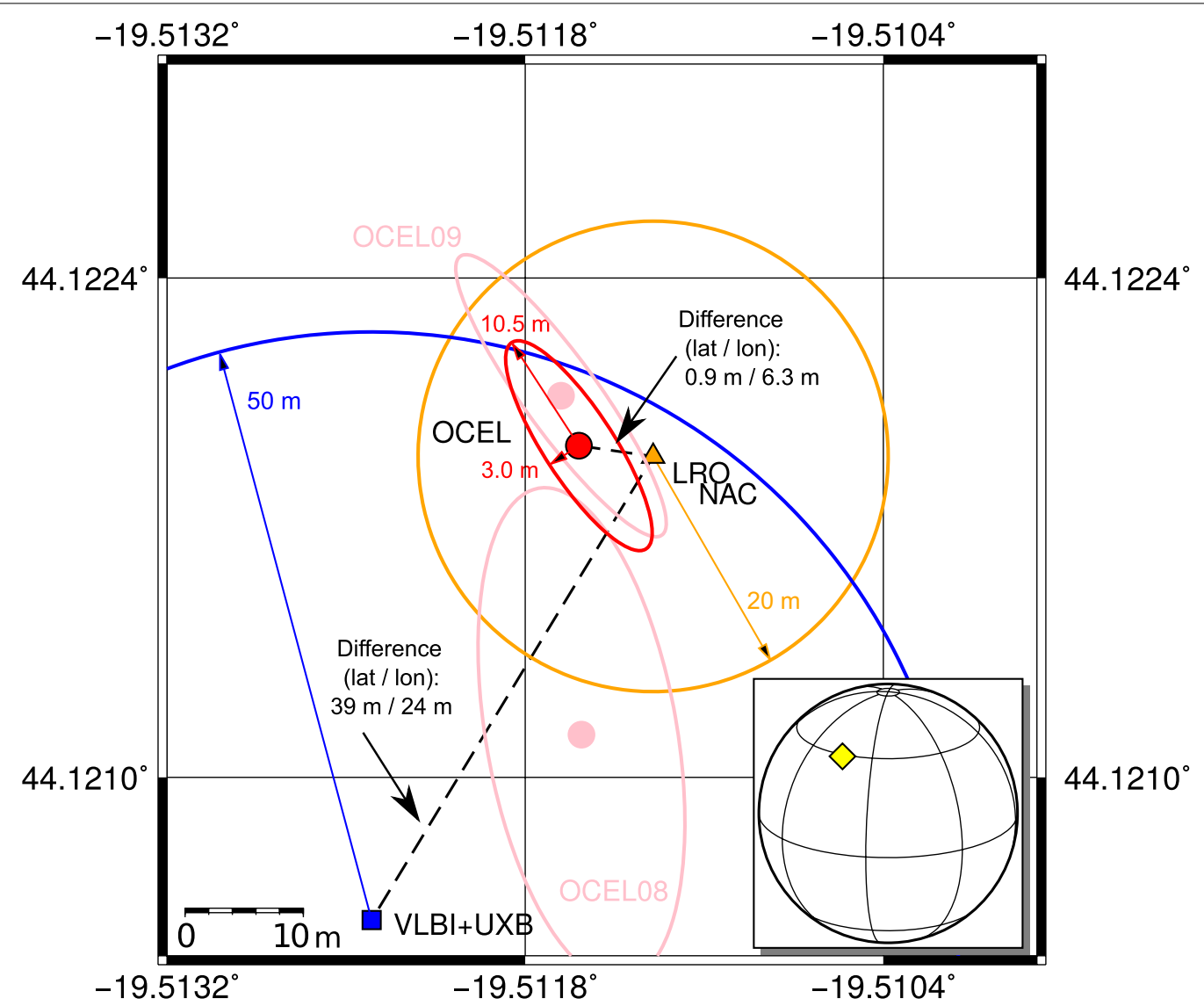

Fig. 3 Comparison of the positions of the CE-3 lander from various studies. The orange triangle refers to the position determined by combining fourteen images taken by the Narrow Angle Camera of the LRO (Liu et al. 2015a). The blue square depicts the coordinates obtained through the use of VLBI and UXB measurements (Li et al. 2014). In both cases, the associated position errors (20 m and $50 \mathrm{~m}$ ) correspond to the uncertainties stated in Liu et al. (2015a) and Li et al. (2014). The pink dots along with the 1- $\sigma$ position error ellipses depict the results obtained from the individual OCEL sessions used in this study. The red dot (with the 1- $\sigma$ error ellipse) represents the weighted mean position of the lander based upon OCELO 8 and OCELO9 
improving the sensitivity of geodetic VLBI for determination of the position of this lunar target (Klopotek et al. 2017b). Compared to VLBI+UXB, the consideration of troposphere and ionosphere delays led to clear improvements of the coordinate estimates and decreased their formal errors.

\section{Conclusions and outlook}

In this paper, the concept of geodetic VLBI observations of the Chang'e 3 lander was presented. We also described the results from the analysis of 24-h VLBI sessions designed for observations of this target using a global network of VLBI telescopes. The analysis was carried out through the combination of quasar and lunar observations in a dedicated VLBI processing strategy. The position of the lander was determined to be $44.12193^{\circ} \mathrm{N}$, $-19.51159^{\circ} \mathrm{E}$ and $-2637.3 \mathrm{~m}$, with the horizontal position uncertainties on the lunar surface of $8.9 \mathrm{~m}$ and $4.5 \mathrm{~m}$ in latitude and longitude, respectively. The derived position differs by about $6 \mathrm{~m}$ from the results from LRO NAC.

OCEL sessions provided first insights concerning global geodetic VLBI observations of artificial lunar radio sources. Based upon the gained knowledge and encountered technical difficulties, organization of similar programs in the future would be valuable to better understand this new observing concept and fully benefit from its true potential (Klopotek et al. 2018). Further steps concern an improvement of the lunar-based parameter determination via an automated and optimized scheduling as well as an enhancement of the postcorrelation analysis of lunar observations. It is expected that the introduction of dual-frequency observations could improve the positioning performance as ionosphere errors would be further reduced and estimation of VTEC biases as well as additional clock offsets could be avoided. Moreover, observations of such targets in the next-generation VLBI system (Niell et al. 2018) would benefit from an improved observation density of both quasar and lunar sources, better handling of tropospheric turbulence as well as significantly decreased observation noise at VLBI stations (Klopotek et al. 2018).

Future work should also include simulation studies concerning measurement sensitivity of geodetic VLBI for determination of parameters related to lunar dynamics or even co-location of VLBI with other space-geodetic techniques via targets on the Moon. With the enhanced measurement precision as well as reduced errors due to the troposphere and ionosphere, the proposed concept could provide scientists with the technique that has the potential to complement Lunar Laser Ranging, e.g., in determination of coordinates of artificial optical and radio targets, leading to a better knowledge on lunar rotation as well as decreased correlations between the estimated coordinates and other lunar-based parameters (Hofmann et al. 2018). Observations of artificial radio sources on the Moon, treated with the approach described in this study, could be incorporated into regular IVS schedules with almost no effort and with no additional or dedicated Earth-based equipment, allowing to observe such lunar targets on a daily basis.

\begin{abstract}
Abbreviations
VLBI: very long baseline interferometry; CE-3: Chang'e 3; NAC: narrow angle camera; IVS: international VLBI service for Geodesy and Astrometry; OCEL: observing the Chang'e 3 lander with VLBI; LRO: lunar reconnaissance orbiter; DOR: differential one-way ranging; ZWD: Zenith wet delay; UXB: unified $X$ band; GIM: global lonospheric Maps; IONEX: IONosphere map EXchange format; VTEC: vertical total electron content; JPL DE: jet propulsion laboratory development ephemeris; WRMS: weighted root mean square; EOP: Earth orientation parameters; IERS: international Earth rotation and reference systems service; RFI: radio frequency interference; ITRF: international terrestrial reference frame.
\end{abstract}

\section{Authors' contributions}

$\mathrm{RH}, \mathrm{ANe}$ and CP performed important initial tests to prepare for the OCEL sessions. RH scheduled the OCEL sessions. LLP and FJ correlated all the data and fringe fitted the quasar (LLP) and lunar observations (FJ). ANo, FJ, ZZ and $\mathrm{SH}$ helped with the interpretation of raw lunar data. GK and TH analyzed both data sets and determined lunar-based parameters. GK wrote the manuscript and created the figures. TH and RH supervised GK and along with other authors helped to improve the manuscript. All authors read and approved the final manuscript.

\section{Author details}

${ }^{1}$ Department of Space, Earth and Environment, Onsala Space Observatory, Chalmers University of Technology, Onsala, Sweden. ${ }^{2}$ Institute of Navigation, University of Stuttgart, Stuttgart, Germany. ${ }^{3}$ Institute of Geodesy and Geoinformation, University of Bonn, Bonn, Germany. ${ }^{4}$ Reichert GmbH, Bonn, Germany. ${ }^{5}$ Beijing Aerospace Control Center, National Key Laboratory of Science and Technology on Aerospace Flight Dynamics, Beijing, China. ${ }^{6}$ Geodetic

Observatory Wettzell, Technical University of Munich, Bad Kötzting, Germany.

${ }^{7}$ Geodetic Observatory Wettzell, Federal Agency for Cartography and Geodesy, Bad Kötzting, Germany.

\section{Acknowledgements}

The aforementioned sites of the IVS and Chinese VLBI networks are acknowledged for their participation in this research project. We thank the reviewers for their constructive comments.

\section{Competing interests}

The authors declare that they have no competing interests.

\section{Availability of data and materials}

The datasets used during the current study are available from the corresponding author on a reasonable request.

Funding

Not applicable.

\section{Publisher's Note}

Springer Nature remains neutral with regard to jurisdictional claims in published maps and institutional affiliations.

Received: 7 December 2018 Accepted: 14 February 2019

Published online: 27 February 2019 


\section{References}

Altamimi Z, Rebischung P, Métivier L, Collilieux X (2016) ITRF2014: a new release of the International Terrestrial Reference Frame modeling nonlinear station motions. J Geophys Res Solid Earth 121:6109-6131

Archinal BA, A'Hearn MF, Bowell E, Conrad A, Consolmagno GJ, Courtin R, Fukushima T, Hestroffer D, Hilton JL, Krasinsky GA, Neumann G, Oberst J, Seidelmann PK, Stooke P, Tholen DJ, Thomas PC, Williams IP (2011) Report of the IAU working group on cartographic coordinates and rotational elements: 2009. Celest Mech Dyn Astron 109(2):101-135

Bizouard C, Gambis D (2018) International Earth Rotation and Reference Systems Service Earth Orientation Parameters EOP (IERS) 14 C04. ftp://hpier s.obspm.fr/iers/eop/eopc04/. Accessed 2552018

Bolotin S, Baver K, Gipson J, Gordon D, MacMillan D (2014) The VLBI data analysis software vSolve: development progress and plans for the future. In: Baver KD, Behrend D, Armstrong KL (eds) IVS 2014 general meeting proceedings. Science Press, Beijing, pp 253-257

Cao J, Zhang Y, Hu S, Huang Y, Chen M (2016) An analysis of precise positioning and accuracy of the CE-3 lunar lander soft landing. Geomat Inf Sci Wuhan Univ 41(2):274 (In Chinese)

Deller AT, Brisken WF, Phillips CJ, Morgan J, Alef W, Cappallo R, Middelberg E, Romney J, Rottmann H, Tingay SJ, Wayth R (2011) DiFX-2: a more flexible, efficient, robust, and powerful software correlator. Publ Astron Soc Pac 123(901):275

Duev DA, Calves MG, Pogrebenko SV, Gurvits LI, Cimo G, Bahamon TB (2012) Spacecraft VLBI and doppler tracking: algorithms and implementation. Astron Astrophys 541:A43

Elphic RC, Delory GT, Hine BP, Mahaffy PR, Horanyi M, Colaprete A, Benna M, Noble SK (2014) The Lunar atmosphere and dust environment explorer mission. Space Sci Rev 185(1):3-25

Fey AL, Gordon D, Jacobs CS (eds) (2009) The second realization of the international celestial reference frame by very long baseline interferometry, Presented on behalf of the IERS/IVS Working Group. IERS Technical Note 35, Verlag des Bundesamts für Kartographie und Geodäsie, Frankfurt am Main

Folkner WM, Williams JG, Boggs DH (2009) The planetary and lunar ephemeris DE 421. IPN progress report 42-178

Haas R, Halsig S, Han S, Iddink A, Jaron F, La Porta L, Lovell J, Neidhardt A, Nothnagel A, Plötz C, Tang G, Zhang Z (2017) Observing the Chang'E-3 Lander with VLBI (OCEL): Technical setups and first results. In: Nothnage A, Jaron $F$ (eds) Proceedings of the first international workshop on VLBI observations of near-field targets, October 5-6, 2016, Schriftenreihe des Inst. f. Geodäsie u. Geoinformation, Vol 54, ISSN 1864-1113, Bonn, vol 54 pp 41-64

Hellerschmied A, McCallum L, McCallum J, Sun J, Böhm J, Cao J (2018) Observing APOD with the AuScope VLBI array. Sensors 18(5):1587

Hobiger T, Otsubo T (2017) Combination of space geodetic techniques on the observation level with c5++: common nuisance parameters and data weighting. In: van Dam T (ed) REFAG 2014. Springer, Cham, pp 31-37

Hobiger T, Kondo T, Schuh H (2006) Very long baseline interferometry as a tool to probe the ionosphere. Radio Sci 41(1):1-10

Hobiger T, Otsubo T, Sekido M, Gotoh T, Kubooka T, Takiguchi H (2010) Fully automated VLBI analysis with c5++ for ultra rapid determination of UT1. Earth Planets Space 62(12):933-937

Hofmann F, Biskupek L, Müller J (2018) Contributions to reference systems from Lunar Laser Ranging using the IfE analysis model. J Geod 92(9):975-987

Jones DL, Folkner WM, Jacobson RA, Jacobs CS, Dhawan V, Romney J, Fomalont E (2015) Astrometry of Cassini With the VLBA to improve the Saturn Ephemeris. Astron J 149:28
Kato M, Sasaki S, Tanaka K, lijima Y, Takizawa Y (2008) The Japanese lunar mission SELENE: science goals and present status. Adv Space Res 42(2):294-300

Klopotek G, Hobiger T, Haas R (2017a) Implementation of VLBI Near-Field Delay Models in the c5++ Analysis Software. In: Nothnagel A, Jaron F (eds) Proceedings of the first international workshop on VLBI observations of near-field targets, October 5-6, 2016, Schriftenreihe des Inst. f. Geodäsie u. Geoinformation, Vol 54, ISSN 1864-1113, Bonn, vol 54, pp 29-33

Klopotek G, Hobiger T, Haas R (2017b) Lunar Observations and Geodetic VLBI-A simulation study. In: Haas R, Elgered G (eds) Proceedings of the 23rd European VLBI group for Geodesy and Astrometry working meeting, Chalmers University of Technology, Gothenburg, pp 122-126

Klopotek G, Hobiger T, Haas R (2018) Geodetic VLBI with an artificial radio source on the Moon: a simulation study. J Geod 92(5):457-469

Li C, Liu J, Ren X, Zuo W, Tan X, Wen W, Li H, Mu L, Su Y, Zhang H, Yan J, Ouyang $Z$ (2015) The Chang'e 3 mission overview. Space Sci Rev 190(1):85-101

Li P, Huang Y, Chang S, Hu X, Liu Q, Zheng X, Wang G, Zheng W, Fan M (2014) Positioning for the Chang'E-3 lander and rover using earth-based observations. Chin Sci Bull 59(32):3162 (In Chinese)

Liu B, Di K, Wang B, Tang G, Xu B, Zhang L, Liu Z (2015a) Positioning and precision validation of Chang'E-3 lander based on multiple LRO NAC images. Chin Sci Bull 60(28-29):2750 (In Chinese)

Liu Z, Di K, Peng M, Wan W, Liu B, Li L, Yu T, Wang B, Zhou J, Chen H (2015b) High precision landing site mapping and rover localization for Chang'e-3 mission. Sci China Phys Mech Astron 58(1):1-11 (In Chinese)

Lonsdale CJ (1996) Haystack Observatory Postprocessing System (HOPS). ftp:// gemini.haystack.mit.edu/pub/hops/what_is_hops. Accessed 2562018

Niell A, Barrett J, Burns A, Cappallo R, Corey B, Derome M, Eckert C, Elosegui P, McWhirter R, Poirier M, Rajagopalan G, Rogers AEE, Ruszczyk C, SooHoo J, Titus M, Whitney A, Behrend D, Bolotin S, Gipson J, Gordon D, Himwich E, Petrachenko B (2018) Demonstration of a broadband very long baseline interferometer system: a new instrument for high-precision space Geodesy. Radio Sci 53(10):1269-1291

Nothnagel A, Artz T, Behrend D, Malkin Z (2017) International VLBI service for Geodesy and Astrometry - delivering high-quality products and embarking on observations of the next generation. J Geod 91(7):711-721

Petit G, Luzum B, (eds) (2010) IERS Conventions, (2010) IERS Technical Note 36. Verlag des Bundesamts für Kartographie und Geodäsie, Frankfurt am Main

Schaer S, Beutler G, Rothacher M, Springer TA (1996) Daily global ionosphere maps based on GPS carrier phase data routinely produced by the CODE. In: Neilan RE, Van Scoy PA, Zumberge JF (eds) Proceedings of the IGS Analysis Center Workshop, International GNSS Service

Sekido M, Kondo T, Kawai E, Imae M (2003) Evaluation of GPS-based ionospheric TEC map by comparing with VLBI data. Radio Sci 38(4)

Sovers OJ, Fanselow JL, Jacobs CS (1998) Astrometry and Geodesy with radio interferometry: experiments, models, results. Rev Mod Phys 70(4):1393-1454

Tooley CR, Houghton MB, Saylor RS, Peddie C, Everett DF, Baker CL, Safdie KN (2010) Lunar reconnaissance orbiter mission and spacecraft design. Space Sci Rev 150(1):23-62

Zhou H, Li H, Dong G (2015) Relative position determination between Chang'E-3 lander and rover using in-beam phase referencing. Sci China Inf Sci 58(9):1-10 